\title{
BMJ Open Epidemiology of rubella infection in Cameroon: a 7-year experience of measles and rubella case-based surveillance, 2008-2014
}

\author{
Marcellin Nimpa Mengouo, ${ }^{1}$ Valantine Ngum Ndze, ${ }^{2,3}$ Frangy Baonga, ${ }^{4}$ \\ Marie Kobela, ${ }^{4}$ Charles Shey Wiysonge ${ }^{5}$
}

To cite: Nimpa Mengouo M, Ndze VN, Baonga F, et al. Epidemiology of rubella infection in Cameroon: a 7-year experience of measles and rubella case-based surveillance, 2008-2014. BMJ Open 2017;7:e012959. doi:10.1136/ bmjopen-2016-012959

- Prepublication history and additional material is available. To view please visit the journal (http://dx.doi.org/10.1136/ bmjopen-2016-012959).

Received 8 June 2016 Revised 1 March 2017 Accepted 9 March 2017

CrossMark

${ }^{1}$ Immunization, Vaccines and Emergency, World Health Organization Cameroon Country Office, Yaounde, Cameroon ${ }^{2}$ Centre for Evidence Based Health Care, Faculty of Medicine and Health Sciences, Stellenbosch University, Cape Town, South Africa

${ }^{3}$ Department of Microbiology, Haematology, Parasitology and Infectious Disease, Faculty of Medicine and Biomedical Sciences, University of Yaounde I, Yaounde, Cameroon ${ }^{4}$ Expanded Program on Immunization, Ministry of Public Health, Yaounde, Cameroon

${ }^{5}$ Cochrane South Africa, South African Medical Research Council, Tygerberg, South Africa

Correspondence to Dr Valantine Ngum Ndze; valentinengum@yahoo.com

\section{ABSTRACT}

Objective The aim of this study was to estimate the proportion of rubella disease in a measles case-based surveillance in Cameroon prior to rubella vaccine introduction into the national immunisation programme. Design This was a cross-sectional study for rubella infection in Cameroon for the period 2008 to 2014.

Setting Patients suspected with measles from the 10 regions of Cameroon were recruited according to the WHO measles case definition and were tested for rubella IgM antibodies accompanied with the case report/investigation forms.

Participants All persons with rash and fever within 14 days of onset of rash according to the standard WHO African Regional Office (WHO/AFRO) case definition for a suspected measles case.

Outcome measures Descriptive analyses and simple logistic regressions were performed. OR were estimated. Results A total of 9907 serum samples from people with fever and rash were received in the laboratory from 2008 to 2014. A total of $7489(75.59 \%)$ measles-negative samples were tested for rubella; 699 (9.3\%) were positive for rubella IgM antibodies. Logistic regression analysis was done using IgM antibodies detection as the outcome variable. Age, sex and setting were explanatory variables. Logistic regression analysis revealed that, comparing the proportion of rubella IgM seropositivity status by age, the association to a positive rubella IgM increased with age from 1 to 4 years (OR $7.11 ; 95 \% \mathrm{Cl} 4.35$ to 12.41 ; $\mathrm{p}<0.0001$ ), through 5 to 9 years (OR $13.07 ; 95 \% \mathrm{Cl}$ 7.93 to $22.93 ; p<0.001)$, to 10 to 14 years of age (0R 13.86; $95 \% \mathrm{Cl} 8.06$ to 25.12; $p<0.001)$. Persons aged $\geq 15$ years were also more likely to have rubella infection than children under one (OR 3.69; $95 \% \mathrm{Cl} 1.85$ to 7.48 ; $\mathrm{p}=0.0001$ ). There were also significant associations with sex, with males being less associated to a positive rubella serology than females $(0 \mathrm{R} 1.33 ; 95 \% \mathrm{Cl} 1.14$ to $1.56 ; p=0.0001)$. No statistically significant difference in proportion of rubella cases was observed between urban and rural populations (OR 1.11; $95 \% \mathrm{Cl} 0.94$ to 1.31 ; $\mathrm{p}=0.208$ ).

Conclusions This study reveals that rubella virus circulates in Cameroon, with important number of cases in children under 15 years. This finding supports the planned introduction of rubella-containing vaccines into the Expanded Program on Immunization.

\section{Strengths and limitations of this study}

- This is the first study in Cameroon to investigate rubella infection across a broad age range, from all the regions and investigating association with some sociodemographic factors.

- The integration of rubella testing with measles casebased surveillance provides an opportunity to gain understanding about the epidemiology of rubella infection.

- The sensitivity of the case definition used for measles surveillance is likely not high enough to identify all rubella cases.

- The existence of missing data for some variables highlights some issues in data management process.

\section{INTRODUCTION}

Rubella is an acute, contagious viral disease caused by a Togavirus of the genus Rubivirus and is characterised by a mild maculopapular rash. ${ }^{1}$ The illness is often mild in children but has serious consequences in pregnant women. Arthralgia or arthritis may occur in up to $70 \%$ of adult women infected with rubella with rare complications including thrombocytopenic purpura and encephalitis. ${ }^{23}$ The infection in women during the first trimester of pregnancy can severely affect the fetus, resulting in miscarriage, fetal death or the combination of disabling conditions collectively called congenital rubella syndrome (CRS). Common birth defects are ocular (retinitis, microphthalmia, glaucoma and cataracts), hearing impairment, hearing defects, pulmonary stenosis, persistent ductus arteriosus, microcephaly, developmental delay, mental retardation, bone alterations and damage to the liver and spleen. ${ }^{4}$ Global estimates of the burden of rubella suggest that 100000 infants are born with CRS each year which makes rubella a leading cause of preventable 
congenital defects. ${ }^{56}$ The estimates for 2008 suggest that the highest CRS burden is in South-East Asia (48\%) and Africa (38\%). Economic analysis for rubella and CRS control suggest that incorporation of rubella-containing vaccine (RCV) into national childhood immunisation schedules is both cost-beneficial and cost-effective. ${ }^{7}$ Estimates suggest a wide range for the lifetime cost of treating a single CRS case from US $\$ 4200$ (inflation-adjusted 2012), in a middle-income country such as Brazil, to over US\$200000 (inflation-adjusted 2012), in a high-income country such as the USA. ${ }^{8}$ At least five of six WHO regions have set rubella control or elimination targets by the year 2020, since the Measles and Rubella Initiative was launched in $2001 .^{7}$

In Cameroon, the national Expanded Program on Immunization (EPI) was established in 1976 and includes provision of the first dose of measles-containing vaccine (MCV1) at 9 months of age. Measles case-based surveillance has been integrated to the acute flaccid paralysis surveillance system to monitor the progress with measles control. Rubella vaccination has not yet been introduced into the infant vaccination schedule in Cameroon, and furthermore, surveillance of rubella per se or CRS does not exist. However, the measles case-based surveillance system, established in 2004, includes laboratory testing for the detection of measles-specific and rubella-specific IgM antibodies. Cameroon planned to introduce the (RCV) into the EPI in 2016 starting by a national measles and rubella mass vaccination campaign for children under 15 years. WHO recommends that epidemiological analysis should be carried out in the process of rubella vaccine introduction to evaluate the burden of the disease. ${ }^{9}$ However, very limited studies have been conducted in the country to determine the epidemiology of the disease and gather baseline evidence prior to this introduction.

This study aims to determine the epidemiology of rubella disease in Cameroon based on the national measles case-based surveillance system from 2008 to 2014. The outcome of this analysis provides baseline data for future monitoring of the impact of rubella vaccines in the national EPI programme.

\section{METHODS}

\section{Sampling and laboratory procedure}

We carried out a cross-sectional study on the national rubella case-based surveillance activities for the period 2008 to 2014 in Cameroon. Cameroon is located in Central Africa within the WHO African Region with a projected population of 22179707 habitants in 2015. It was estimated that children under 5 years account for about $16.6 \%$ and about $51 \%$ of the population were female. ${ }^{10}$ Life expectancy at birth is estimated to be 58 years, and the total fertility rate is six children per woman of childbearing age. ${ }^{11}$

During the study period, blood samples were collected by nurses from persons with rash and fever within 14 days of onset of rash according to the standard WHO African Regional Office (WHO/AFRO) case definition for a suspected measles case (ie, any person with generalised maculopapular rash and fever plus one of the following: cough or coryza (runny nose) or conjunctivitis (red eyes) or any person in whom a clinician suspects measles). This case definition was applied to both children and adults seeking care in health facilities all over the country. According to the WHO guidelines for measles case confirmation, suspected cases were tested for the presence of measles IgM antibodies. ${ }^{12}$ However, in the case of a measles outbreak, only five suspected cases are tested for the presence of measles IgM antibodies and if three or more of the cases test measles IgM positive, this is declared as a laboratory-confirmed outbreak. In the confirmed outbreak situation, no further samples are collected in the affected area until 30 days after the initial confirmation of the outbreak.

At first contact with a suspected measles case, about $1-5 \mathrm{~mL}$ of blood was collected by venipuncture into a sterile anticoagulant-free tube. The blood was then allowed to clot then centrifuged at $3000 \mathrm{rpm}$ for $5 \mathrm{~min}$ to separate the serum. If there was no centrifuge in the health centre, the blood was stored in a refrigerator until the clot was completely retracted from the serum. The serum was transferred aseptically into a phial and stored at $2^{\circ} \mathrm{C}-8^{\circ} \mathrm{C}$ for at most 3 days before being transported in a cold chain to Pasteur Institute Cameroon, which houses the national measles/rubella surveillance laboratory, accredited by the WHO Global Measles and Rubella Laboratory Network (Labnet).

In the testing laboratory, sera were stored at $-20^{\circ} \mathrm{C}$ and tested within 7 days. ELISA for rubella-specific IgM antibodies was carried out according to the manufacturer's instructions (Enzygnost Anti-Rubella Virus/IgM kit; Siemens, Erlangen, Germany). Patients with rubella IgM-positive serum samples were classified as laboratory-confirmed rubella cases. Due to limited resources, all samples were tested once and those producing equivocal results were not retested.

\section{Data management and analysis}

The laboratory results and completed individual case investigation forms were sent to the EPI programme. Forms collected from the laboratory were reviewed and entered into the WHO electronic database. The casebased surveillance data were reported on a weekly basis by the EPI programme to the WHO country office.

Statistical analyses were performed using the SPSS statistical package (V.16). Data were cleaned and analysis of rubella cases done by year, age, age group, sex, setting/region and months. Descriptive analysis was completed with bivariate analyses using ${ }^{2}$ or Fisher's exact tests for categorical variables to find key determinant of rubella infection in Cameroon. A 95\% CI was calculated for the OR, and values of $p<0.05$ were considered statistically significant. Logistic regression analysis was used to estimate OR of IgM seropositivity 
Table 1 Proportion of rubella cases in Cameroon by year (2008-2014)

\begin{tabular}{llll}
\hline Year & & \multicolumn{2}{l}{ Outcome of tests } \\
\cline { 3 - 4 } & $\begin{array}{l}\text { Number tested } \\
\text { for rubella IgM }\end{array}$ & Positive (\%) & Indeterminate (\%) \\
\hline 2008 & 646 & $78(12.1 \%)$ & $20(3.1 \%)$ \\
2009 & 688 & $32(4.7 \%)$ & $1(0.1 \%)$ \\
2010 & 655 & $42(6.4 \%)$ & $6(0.9 \%)$ \\
2011 & 1107 & $105(9.5 \%)$ & $30(2.7 \%)$ \\
2012 & 1185 & $147(12.4 \%)$ & $43(3.6 \%)$ \\
2013 & 1342 & $147(11.0 \%)$ & $44(3.3 \%)$ \\
2014 & 1866 & $148(7.9 \%)$ & $58(3.1 \%)$ \\
Total & 7489 & $699(9.3 \%)$ & $202(2.7 \%)$ \\
\hline
\end{tabular}

as outcome variable with age, sex and setting as explanatory variable.

\section{RESULTS}

\section{Proportion of rubella diseases}

A total of 9907 samples from suspected measles cases were received in the laboratory from 2008 to 2014 among which $7489(75.59 \%)$ measles-negative samples were tested for rubella; $699(9.3 \%)$ were positive for rubella IgM antibodies. The proportion of rubella IgM antibodies varied from $4.7 \%$ in 2009 to $12.4 \%$ in 2012 (table 1). Also, results of $202(2.7 \%)$ cases were indeterminate and were treated as negative.

\section{Distribution of rubella cases by age}

Among the 699 positive cases, the median age was 5 years, range 5 months to 68 years. Figure 1 shows that $2.43 \%$ of rubella-positive cases were children below 1 year of age (representing the targeted age in the routine EPI programme) and $96.85 \%$ of cases were below 15 years (marking the entrance to childbearing age for young girls).

\section{Rubella distribution by age group}

The proportion by age group shows that the age groups between 5-9 years and 10-14 years had highest proportions of rubella infection (figure 1 and table 2).

\section{Distribution of rubella cases by sex}

The sex was available for 698 cases $(99.85 \%)$ out of 699 . More than half $(380 / 698,54.44 \%)$ of positive rubella cases were females. Females were 1.3 times more associated to a positive rubella IgM than males (OR 1.33; 95\% CI 1.14 to $1.56 ; \mathrm{p}=0.0001$ ) (table 2 ).

\section{Rubella in women of childbearing age (15-49 years)}

There were 242 females of childbearing age who were tested, $11(4.55 \%)$ were positive for rubella IgM antibodies. The proportion of positive rubella cases within this high-risk group varied from one year to another, with the lowest of $0.0 \%$ in 2008 and the highest of $15.8 \%$ in 2012.

\section{Rubella case distribution by setting/region}

The setting was specified for 7466 suspected rubella cases $(99.7 \%)$ out of 7489 . There was no statistically significant difference in proportion of rubella cases between urban and rural populations (OR 1.11; 95\% CI 0.94 to $1.31 ; \mathrm{p}=0.208$ ) (table 2 ). The proportion of rubella-positive cases by regions in Cameroon between 2008 and 2014 shows that, the East, North-West and the SouthWest regions had higher proportions of rubella cases (figure 2).

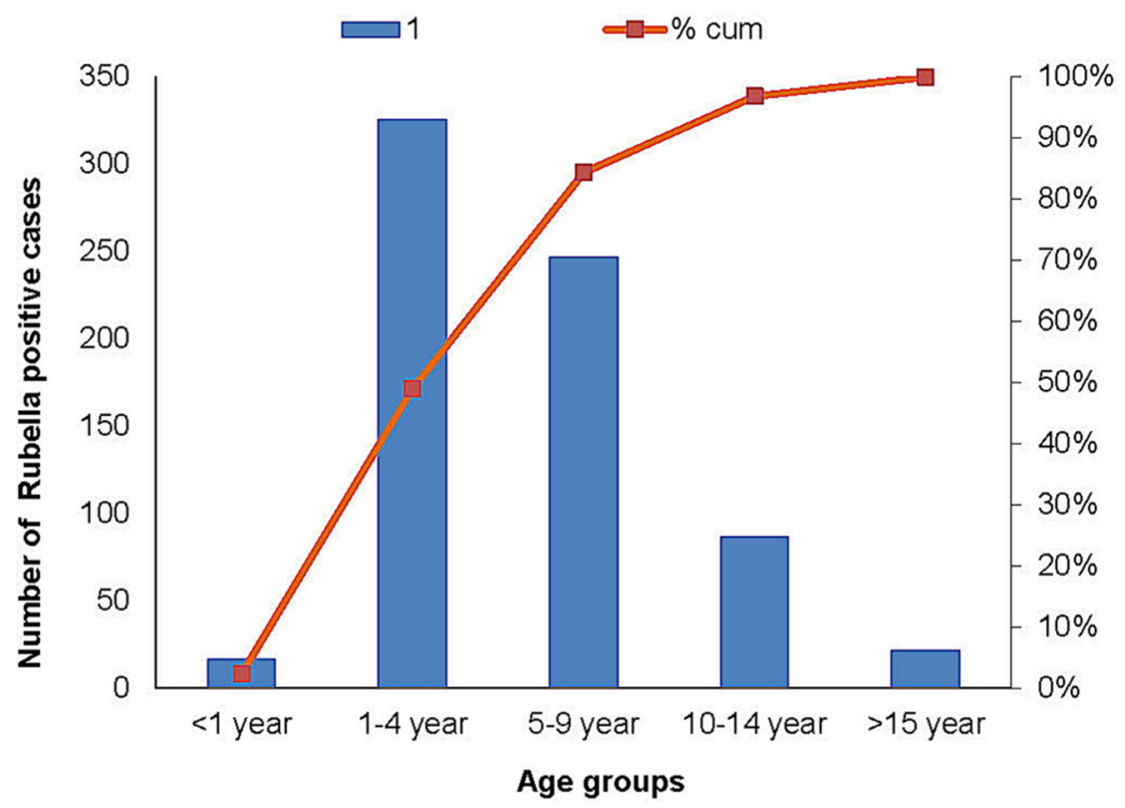

Figure 1 Rubella case distribution by age in Cameroon, 2008-2014. 
Table 2 Distribution of rubella cases by some sociodemographic characteristics in Cameroon, 2008-2014

\begin{tabular}{|c|c|c|c|c|c|}
\hline & Number tested for rubella & Number tested positive (\%) & OR & $95 \% \mathrm{Cl}$ & $P$ value \\
\hline \multicolumn{6}{|l|}{ Age group } \\
\hline$<1$ year & 1244 & $17(1.37)$ & 1 & & 0.0001 \\
\hline $5-9$ years & 1610 & 247 (15.34) & 13.07 & 7.93 to 22.93 & \\
\hline $10-14$ years & 540 & $87(16.11)$ & 13.86 & 8.06 to 25.12 & \\
\hline Total & 7481 & $699(9.3)$ & & & \\
\hline \multicolumn{6}{|l|}{$\operatorname{Sex}$} \\
\hline Male & 3904 & $318(8.2)$ & 1 & & 0.0001 \\
\hline Female & 3584 & $380(10.6)$ & 1.33 & 1.14 to 1.56 & \\
\hline Total & 7488 & $698(9.3)$ & & & \\
\hline \multicolumn{6}{|l|}{ Setting } \\
\hline Urban & 2651 & $264(9.9)$ & 1.11 & 0.94 to 1.31 & \\
\hline Total & 7466 & $699(9.3)$ & & & \\
\hline
\end{tabular}

\section{Seasonal distribution of rubella cases from 2008 to 2014}

The analysis of the number of rubella cases during the study period 2008 to 2014 revealed an apparent seasonality in Cameroon. Monthly variation showed that the number of rubella cases during the months of February to April was highest, which represents the end of the dry season and beginning of the raining season in most parts of Cameroon with a sharp decline in numbers in the months of July through September (figure 3).

\section{Logistic regression analysis}

Logistic regression analysis reveals associations of rubella IgM seropositivity with age. Compared with children less than 1 year of age, the association increased with age from 1 to 4 years (OR 7.11; 95\% CI 4.35 to 12.41 ; $\mathrm{p}<0.0001$ ), through 5 to 9 years (OR 13.07; 95\% CI 7.93 to $22.93 ; \mathrm{p}<0.001$ ), to 10 to 14 years of age (OR 13.86; $95 \%$ CI 8.06 to 25.12; $\mathrm{p}<0.001)$. In addition, people aged
15 years and above captured in the measles case-based surveillance system were also more likely to have rubella infection than children under one (OR 3.69; 95\% CI 1.85 to $7.48 ; \mathrm{p}=0.0001)$. There were also significant associations with sex, with males being less associated to a positive rubella IgM than females (OR 1.33; 95\% CI 1.14 to $1.56 ; \mathrm{p}=0.0001$ ).

\section{DISCUSSION}

The national measles case-based surveillance system in Cameroon enabled the estimation of the proportion of rubella infections among suspected measles cases. From this study, we observed that during the measles outbreaks between 2008 and 2014, the proportion of rubella cases ranged from $4.7 \%$ in 2009 to $12.4 \%$ in 2012, with number of cases ranging from 32 to 148 per year. The increase in the proportion of rubella cases during this period could

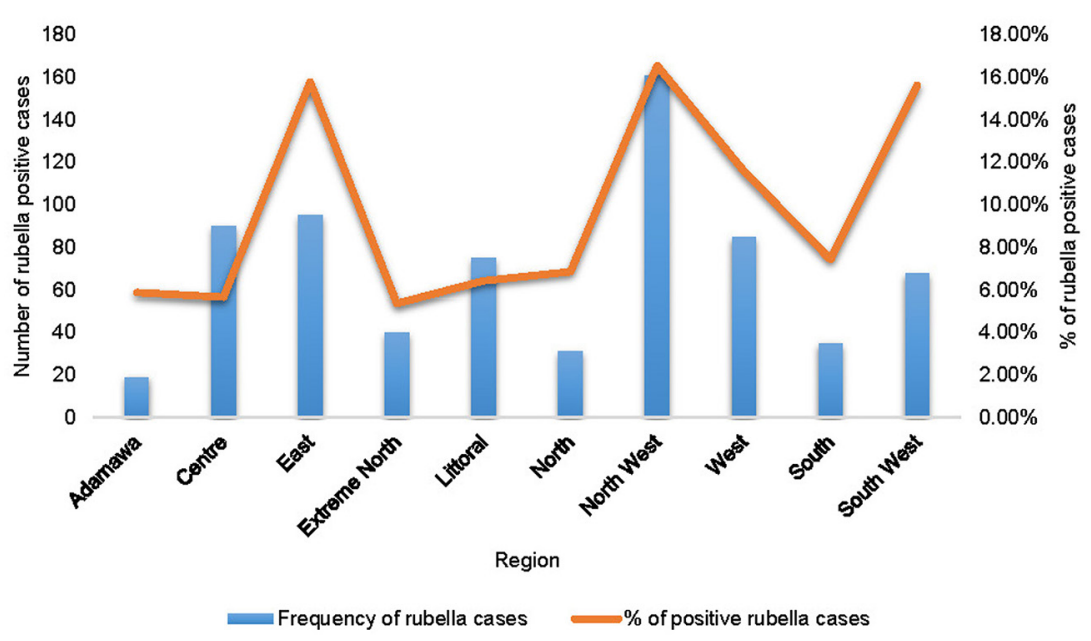

Figure 2 Distribution of rubella-positive cases by region in Cameroon, 2008-2014. 
60

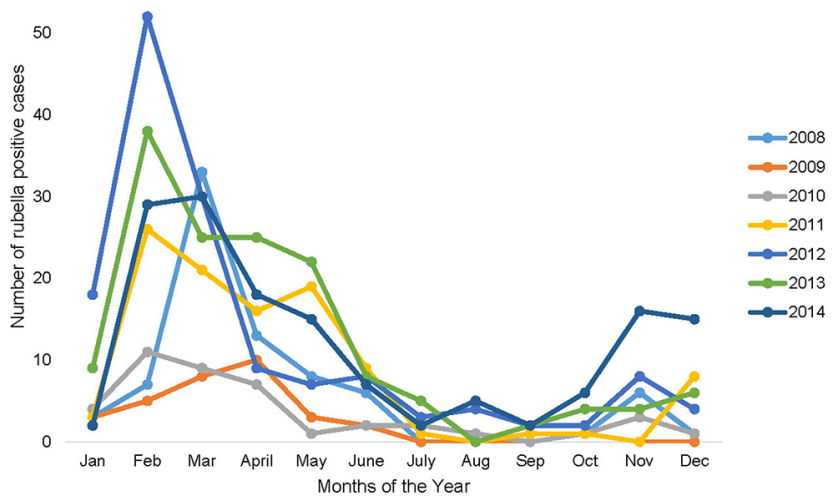

Figure 3 Monthly trends in the number of samples testing positive for rubella in Cameroon, 2008-2014.

actually be indicating a rubella outbreak also, and this type of observation necessitates a clear case definition of rubella infection. Also, the observed increase in rubella cases detected, particularly in 2010 and 2011, could be attributed to the improvements in the sensitivity of the measles case-based surveillance system, through linkage with the strengthened acute flaccid paralysis surveillance system within the context of polio eradication.

Most rubella cases were reported in children below 15 years, indicating that rubella was mainly an illness of young and early-school-age children. This was similar to findings in Ethiopia and Zimbabwe where 94\% and 98\% of cases, respectively, came from this age group. ${ }^{13}{ }^{14}$ Similarly, the lowest proportion of cases was seen in children below 1 year thereby substantiating the potential benefit if a rubella vaccine is incorporated into the national routine immunisation programme which targets children of this age group. Vaccinating children in the routine immunisation programme at this age will provide protection against rubella in these children through adolescence and adulthood by interrupting virus circulation early in childhood and ideally establishing herd immunity in the population. This will further reduce the risk of acquiring rubella infection during pregnancy and also reduce the burden of CRS.

The proportion of females of reproductive age with positive rubella IgM among captured suspected measles cases was very low $(4.55 \%)$. It has been documented in other regions that, higher rubella IgM seroprevalence rate was observed in children and adolescent than in adults, with the trend of IgM positivity decreasing with increase in age. ${ }^{13-20}$ The apparent association of positive rubella IgM results to females should be interpreted with caution as the small differences could be as a result of diagnostic or selection bias in the women than in the men.

There was an apparent seasonal trend in the number of rubella cases where peaks were seen during the end of the dry season (February) and beginning of the raining season (April) in most parts of Cameroon with a sharp decline in numbers in the month of July through September then gradually begins to increase from October through December. A similar trend was noted by Mitiku et $a l^{13}$ in Ethiopia where a seasonal distribution of cases occurred each year and peaked from March to June. Rubella cases in this study do not show any statistically significant difference between urban and rural populations. Mitiku et al ${ }^{13}$ noted a significantly higher number of positive cases in the urban areas compared with rural areas.

Occurrence of rubella antibodies in children of 5 months in this study indicates possibility of infection at a younger age although CRS burden is still unknown in Cameroon. In a literature review of CRS and acquired rubella in developing countries, Cutts et al reported that data from developing countries suggested that the risk of CRS is at least as high as that in industrialised countries during the prevaccination era. ${ }^{21}$ The incidence of CRS per 1000 live births in Cameroon is still unknown. Jivraj et $a l^{22}$ revealed ocular and auditory manifestations of CRS among 320 students from two schools (one for children with hearing impairment and one for children with normal hearing) in Mbingo, Cameroon with 28 (10.2\%) probable or clinically confirmed cases according to the WHO case definitions. A probable case of CRS was defined as an individual with two or more of the following conditions: cataract, congenital glaucoma, pigmentary retinopathy or hearing impairment which are clinical major signs of rubella infection. Hearing-impaired children were seven times more likely to have positive serology $(48.8 \%)$ than children with normal hearing $(7.4 \% ; \mathrm{p}<0.0001) .^{22}$

These results are subject to some limitations. First, the case definition used to detect the rubella cases was designed for the measles case-based surveillance system. Many individuals with rubella disease may not have rash which occurs in $50 \%-80 \%$ of persons infected with rubella. ${ }^{9}$ The sensitivity of the case definition used for measles surveillance is likely not high enough to identify all rubella cases. The system also relies mostly on health facilities and may have missed some community cases. The database also shows existence of missing data for some variables highlighting some issues in the data management and rapid control applications for some key variables.

However, the integration of rubella testing with measles case-based surveillance provides an opportunity to gain understanding about the epidemiology of rubella infection. To better understand the burden and epidemiology of rubella and CRS, Cameroon may consider integration of both surveillances by adopting a more inclusive case definition for measles and rubella, and also conducting well-designed population base studies on rubella burden. The following definition was used by the Pan American Health Organization for rubella surveillance as the region moved towards an elimination target: a suspected case is one in which a health worker suspects rubella. ${ }^{23}$ The European Centre for Disease Prevention and Control also adopted a modified definition, namely, any person with sudden onset of generalised maculopapular rash and at 
least one of the following: cervical adenopathy, suboccipital adenopathy, postauricular adenopathy, arthralgia or arthritis. ${ }^{24}$ As Cameroon and other African countries work toward introducing RCVs and plan elimination goals, a modified case definition will need to be considered so as to improve detection rates.

Another major issue Cameroon should consider when introducing rubella vaccination is that a high vaccination coverage above $80 \%$ is required to sustain immunity from childhood to older age groups. A coverage below $80 \%$ in children will mean shifting susceptibility to rubella in a good number of unvaccinated children through older age particularly in women of childbearing age, thereby secondarily incrlinkedeasing the risk of CRS. Such a shift in susceptibility to older age groups occurred and was documented in Greece and South Africa. ${ }^{25} 26$

Cameroon should therefore consider establishing a sentinel surveillance for CRS and find additional strategies to fill immunity gaps in older age groups. Information from such studies will be useful in monitoring the impact of introducing a rubella vaccine into the EPI programme.

\section{CONCLUSION}

Rubella virus circulates in Cameroon with an important number of children under 15 years being affected. Children under 1 year presented with low proportions indicating a potential benefit of introducing a rubella vaccine into the national routine immunisation programme, as this will mean a good opportunity to interrupt virus circulation and protect many children through adolescence and adulthood.

To better understand the burden and epidemiology of rubella and CRS, Cameroon should consider including a more inclusive case definition for measles and rubella, establishing sentinel surveillance for CRS and conducting appropriate postvaccine studies once the vaccine is introduced into the national immunisation programme among high-risk susceptible groups of the population. Information from such studies will be useful for rubella elimination strategies in Cameroon.

Acknowledgements The authors gratefully thank the Cameroon Ministry of Health for her commitment in measles and rubella elimination, the WHO country office and WHO/AFRO for supporting the national measles/rubella surveillance programme in Cameroon, National Measles/Rubella Laboratory personnel involved in laboratory testing and all healthcare workers involved in case identification and sample collection at all health institutions of Cameroon.

Contributors MNM conceived the study, data collection, data analysis and preparing the draft manuscript. VNN, CSW, MK and FB contributed in data analysis and writing of the manuscript. All authors reviewed the draft and approved the final manuscript.

Funding Funding for the measles/rubella surveillance is provided by the Ministry of Public Health and WHO. But funding was not received by any of the authors for this publication.

Competing interests None declared.

Patient consent Although written consent or assent form was not required, for the purposes of the national surveillance programme, relevant information was provided and verbal permission obtained before samples were collected from a suspected case.
Ethics approval The Cameroon Measles surveillance is a national programme approved by the Ministry of Public Health and supported by WHO/AFRO as part of the global goal to control and eliminate measles and rubella. Patient information and specimen collection respected the procedures stipulated in the WHO/AFRO measles/ rubella surveillance protocol.

Provenance and peer review Not commissioned; externally peer reviewed.

Data sharing statement The original data can only be accessed by the research investigators working at the Ministry of Public Health Cameroon and the WHO Country Office Cameroon. MNM can be contacted if the de-identified dataset is required.

Open Access This is an Open Access article distributed in accordance with the Creative Commons Attribution Non Commercial (CC BY-NC 4.0) license, which permits others to distribute, remix, adapt, build upon this work non-commercially, and license their derivative works on different terms, provided the original work is properly cited and the use is non-commercial. See: http://creativecommons.org/ licenses/by-nc/4.0/

(C) Article author(s) (or their employer(s) unless otherwise stated in the text of the article) 2017. All rights reserved. No commercial use is permitted unless otherwise expressly granted.

\section{REFERENCES}

1. McLean H, Redd S, Abernathy E, et al. VPD surveillance manual, fifth edition, 2012. https://www.cdc.gov/vaccines/pubs/surv-manual/ chpt14-rubella.pdf

2. Sherman FE, Michaels RH, Kenny FM. Acute encephalopathy (ENCEPHALITIS) COMPLICATING rubella. Report of cases with virologic studies, cortisol-production determinations, and observations at autopsy. JAMA 1965;192:675-81.

3. Bayer WL, Sherman FE, Michaels RH, et al. Purpura in congenital and acquired rubella. N Engl J Med 1965;273:1362-6.

4. Cooper LZ, Alford, C.A., Rubella. In Remington JS, ed. Infectious diseases of the fetus and newborn infant. 6th edn. Philadelphia: Elsevier, Saunders, 2006:894-926.

5. World Health Organisation. Rubella vaccines: WHO position paper. Weekly epidemiological record 2011;29:301-16.

6. Featherstone DA, Rota PA, Icenogle J, et al. Expansion of the global measles and rubella laboratory network 2005-09. J Infect Dis 2011;204(Suppl 1):S491-8.

7. WHO. Global measles and rubella strategic plan: 2012-2020, 2012 http://apps.who.int/iris/bitstream/10665/44855/1/9789241503396_ eng.pdf

8. Babigumira JB, Morgan I, Levin A. Health economics of rubella: a systematic review to assess the value of rubella vaccination. $B M C$ Public Health 2013;13:406.

9. WHO. Introducing rubella vaccine into national immunization programmes, a step-by-step guide. WHO/IVB/15.07 2015 http:// apps.who.int/iris/bitstream/10665/184174/1/9789241549370_eng. pdf

10. Bureau Central des Recensements et des Etudes de Population (BUCREP). Etat et structures de la population du Cameroun; recensement 2005. http://www.statistics-cameroon.org/downloads/ Rapport de presentation 3 RGPH.pdf

11. Statistique INdela (INS). Enquête démographique et de santé et à indicateurs multiples (EDS-MICS 2011), Septembre 2012. http:// www.cnls.cm/docs/Rapport EDS 2011_au Cameroun.pdf

12. WHO Regional Office for Africa. Guidelines for measles surveillance, revised December 2011

13. Mitiku K, Bedada T, Masresha B, et al. The epidemiology of rubella disease in Ethiopia: data from the measles case-based surveillance system. J Infect Dis 2011;204(Suppl 1):S239-42.

14. Chimhuya S, Manangazira P, Mukaratirwa A, et al. Trends of rubella incidence during a 5-year period of case based surveillance in Zimbabwe. BMC Public Health 2015;15:294.

15. Mwambe B, Mirambo MM, Mshana SE, et al. Sero-positivity rate of rubella and associated factors among pregnant women attending antenatal care in Mwanza, Tanzania. BMC Pregnancy Childbirth 2014;14:95

16. Junaid SA, Akpan KJ, Olabode AO. Sero-survey of rubella lgM antibodies among children in Jos, Nigeria. Virol J 2011;8:244-5.

17. Bamgboye AE, Afolabi KA, Esumeh FI, et al. Prevalence of rubella antibody in pregnant women in Ibadan, Nigeria. West Afr J Med 2004;23:245-8

18. Ogbonnaya EC, Chinedum EK, John A, et al. Survey of the seroprevalence of IgM antibodies in pregnant women infected with rubella virus. J Biotechnol Pharm Res 2012;3:10-14. 
19. Pennap G, Amauche G, Ajoge $\mathrm{H}$, et al. Serologic survey of specific rubella virus IgM in the sera of pregnant women in Makurdi, Benue State, Nigeria. Afr J Reprod Health 2009;13:69-73.

20. Onakewhor JU, Chiwuzie J. Seroprevalence survey of rubella infection in pregnancy at the University of Benin Teaching Hospital, Benin City, Nigeria. Niger J Clin Pract 2011;14:140-5.

21. Cutts FT, Robertson SE, Diaz-Ortega JL, et al. Control of rubella and congenital rubella syndrome (CRS) in developing countries, part 1: burden of disease from CRS. Bull World Health Organ 1997; 75:55-68.

22. Jivraj I, Rudnisky CJ, Tambe E, et al. Identification of ocular and auditory manifestations of congenital rubella syndrome in Mbingo. Int J Telemed App/ 2014;2014:1-5.
23. Castillo-Salgado C. Case definitions: measles and rubella. Epidemiological Bulletin / PAHO. 1999;20:10. accessed http://www1.paho.org/english/sha/EB_v20n3.pdf

24. WHO. Surveillance guidelines for measles, rubella and congenital rubella syndrome in the WHO European Region. Copenhagen: WHO Regional Office for Europe, 2012. http://www.euro.who.int/_data/ assets/pdf_file/0018/79020/e93035-2013.pdf

25. Panagiotopoulos T, Georgakopoulou T. Epidemiology of rubella and congenital rubella syndrome in Greece, 1994-2003. Euro Surveill 2004;9:17-19.

26. Robertson SE, Cutts FT, Samuel R, et al. Control of rubella and congenital rubella syndrome (CRS) in developing countries, part 2: vaccination against rubella. Bull World Health Organ 1997;75:69-80. 